\title{
Lattices Generated by Two Orbits of Subspaces under Finite Singular Symplectic Groups
}

\author{
Xuemei Liu \\ College of Science, Civil Aviation University of China, Tianjin 300300, China \\ Correspondence should be addressed to Xuemei Liu; xm-liu771216@163.com
}

Received 29 September 2012; Revised 18 November 2012; Accepted 21 November 2012

Academic Editor: P. G. L. Leach

Copyright (C) 2013 Xuemei Liu. This is an open access article distributed under the Creative Commons Attribution License, which permits unrestricted use, distribution, and reproduction in any medium, provided the original work is properly cited.

In the paper titled "Lattices generated by two orbits of subspaces under finite classical group" by Wang and Guo. The subspaces in the lattices are characterized and the geometricity is classified. In this paper, the result above is generalized to singular symplectic space. This paper characterizes the subspaces in these lattices, classifies their geometricity, and computes their characteristic polynomials.

\section{Introduction}

In the following we recall some definitions and facts on ordered sets and lattices (see [1]).

Let $P$ denote a finite set. A partial order on $P$ is a binary relation $\leq$ on $P$ such that

(1) $a \leq a$ for any $a \in P$.

(2) $a \leq b$ and $b \leq a$ implies $a=b$.

(3) $a \leq b$ and $b \leq c$ implies $a \leq c$.

By a partial ordered set (or poset for short), we mean a pair $(P, \leq)$, where $P$ is a finite set and $\leq$ is a partial order on $P$. As usual, we write $a<b$ whenever $a \leq b$ and $a \neq b$. By abusing notation, we will suppress reference to $\leq$, and just write $P$ instead of $(P, \leq)$.

Let $P$ be a poset and let $R$ be a commutative ring with the identical element. A binary function $\mu(a, b)$ on $P$ with values in $R$ is said to be the Möbius function of $P$ if

$$
\sum_{a \leq c \leq b} \mu(a, c)= \begin{cases}1, & \text { if } a=b, \\ 0, & \text { otherwise. }\end{cases}
$$

For any two elements $a, b \in P$, we say $a$ covers $b$, denoted by $b<\cdot a$, if $b<a$ and there exists no $c \in P$ such that $b<c<a$. An element $m$ of $P$ is said to be minimal, (resp., maximal) whenever there is no element $a \in P$ such that $a<m$, (resp., $a>m$ ). If $P$ has a unique minimal, (resp., maximal) element, then we denote it by 0 , (resp., 1 ) and say that $P$ is a poset with 0 , (resp., 1). Let $P$ be a finite poset with 0 . By a rank function on $P$, we mean a function $r$ from $P$ to the set of all the nonnegative integers such that

(1) $r(0)=0$,

(2) $r(a)=r(b)+1$, whenever $b<\cdot a$.

Let $P$ be a finite poset with 0 and 1 . The polynomial

$$
\chi(P, x)=\sum_{a \in P} \mu(0, a) x^{r(1)-r(a)},
$$

is called the characteristic polynomial of $P$, where $r$ is the rank function of $P$.

A poset $P$ is said to be a lattice if both $a \vee b:=\sup \{a, b\}$ and $a \wedge b:=\inf \{a, b\}$ exist for any two elements $a, b \in P$. Let $P$ be a finite lattice with 0 . By an atom in $P$, we mean an element in $P$ covering 0 . We say $P$ is atomic lattice if any element in $P \backslash\{0\}$ is a union of atoms. A finite atomic lattice $P$ is said to be a geometric lattice if $P$ admits a rank function $r$ satisfying

$$
r(a \wedge b)+r(a \vee b) \leq r(a)+r(b), \quad \forall a, b \in P .
$$

In this section we will introduce the concepts of subspaces of type $(m, s, k)$ in singular symplectic spaces. Notation and terminologies will be adopted from Wan's book [2]

We always assume that

$$
K_{v, l}=\left(\begin{array}{ccc}
0 & I^{(v)} & \\
-I^{(v)} & 0 & \\
& & 0^{(l)}
\end{array}\right) .
$$


Let $F_{q}$ be a finite field with $q$ elements, where $q$ is a prime power, and let $E$ denote the subspace of $F_{q}^{(2 v+l)}$ generated by $e_{2 v+1}, e_{2 v+2}, \ldots, e_{2 v+l}$, where $e_{i}$ is the row vector in $F_{q}^{(2 v+l)}$ whose ith coordinate is 1 and all other coordinates are 0 .

The singular symplectic group of degree $2 v+l$ over $F_{q}$, denoted by $S p_{2 v+l, 2 v}\left(F_{q}\right)$, consists of all $(2 v+l) \times(2 v+l)$ nonsingular matrices $T$ over $F_{q}$ satisfying $T K_{l} T^{t}=K_{l}$. The row vector space $F_{q}^{(2 v+l)}$ together with the right multiplication action of $S p_{2 v+l, 2 v}\left(F_{q}\right)$ is called the $(2 v+l)$-dimensional singular symplectic space over $F_{q}$. An $m$-dimensional subspace $P$ in the $(2 v+l)$-dimensional singular symplectic space is said to be of type $(m, s, k)$, if $P K_{l} P^{t}$ is of rank $2 s$ and $\operatorname{dim}(P \cap$ $E)=k$. In particular, subspaces of type $(m, 0,0)$ are called $m$-dimensional totally isotropic subspaces. Clearly, singular symplectic group $S p_{2 v+l, 2 v}\left(F_{q}\right)$ is transitive on the set of all subspaces of the same type in $F_{q}^{(2 v+l)}$, see [2, Theorems 3.22].

The results on the lattices generated by one orbit of subspaces under finite classical groups may be found in Gao and You [3], Huo et al. [4-6], Huo and Wan [7], Orlik and Solomon [8], Wang and Feng [9], Wang and Guo [10], and Wang and $\mathrm{Li}[11]$.

For $1 \leq m_{1} \leq m_{2} \leq 2 v-1,0 \leq k_{1} \leq k_{2} \leq l$, let $L_{1}\left(m_{1}, s_{1}, k_{1} ; 2 v+l, v\right)$, (resp., $\left.L_{2}\left(m_{2}, s_{2}, k_{2} ; 2 v+l, v\right)\right)$ denote the set of all subspaces which are sums (resp. intersections) of subspaces in $M\left(m_{1}, s_{1}, k_{1} ; 2 v+l, v\right)$, (resp., $M\left(m_{2}, s_{2}, k_{2} ; 2 v+\right.$ $l, v))$ such that $M\left(m_{2}, s_{2}, k_{2} ; 2 v+l, v\right) \subseteq L_{1}\left(m_{1}, s_{1}, k_{1} ; 2 v+\right.$ $l, v)$, (resp., $\left.M\left(m_{1}, s_{1}, k_{1} ; 2 v+l, v\right) \subseteq L_{2}\left(m_{2}, s_{2}, k_{2} ; 2 v+l, v\right)\right)$. Suppose $L\left(m_{1}, s_{1}, k_{1} ; m_{2}, s_{2}, k_{2} ; 2 v+l, v\right)$ denotes the intersection of $L_{1}\left(m_{1}, s_{1}, k_{1} ; 2 v+l, v\right)$ and $L_{2}\left(m_{2}, s_{2}, k_{2} ; 2 v+l, v\right)$ containing 0 and $F_{q}^{(2 v+l)}$. By ordering $L\left(m_{1}, s_{1}, k_{1} ; m_{2}, s_{2}, k_{2} ; 2 v+\right.$ $l, v)$ by ordinary or reverse inclusion, two families of atomic lattices are obtained, denoted by $L_{O}\left(m_{1}, s_{1}, k_{1} ; m_{2}, s_{2}, k_{2} ; 2 v+\right.$ $l, v)$ or $L_{R}\left(m_{1}, s_{1}, k_{1} ; m_{2}, s_{2}, k_{2} ; 2 v+l, v\right)$, respectively. Wang and Guo [12] discussed the geometricity of the two lattices when $l=0$. In this paper, we generalized their result to general case, characterizes the subspaces in these lattices in Section 2, classify their geometricity in Section 3, and computes their characteristic polynomials in Section 4.

\section{Characterization of Subspaces Contained in $L\left(m_{1}, s_{1}, k_{1} ; m_{2}, s_{2}, k_{2} ; 2 v+l, v\right)$}

Lemma 1. Let $2 v+l>0,0 \leq k \leq l, 2 s \leq m-k \leq v+s$ and $m-k \geq 1$. For any subspace $P$ of type $(m+1, s, k+1)$, there are two subspaces $P_{1}$ and $P_{2}$ of type $(m, s, k)$ such that $P=P_{1}+P_{2}$.

Proof. Assume that

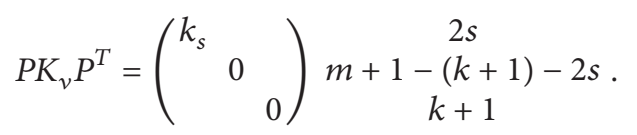

Write $P=\left(\begin{array}{cc}P_{11} & P_{12} \\ 0 & P_{22} \\ 2 v & l\end{array}\right)_{k+1}^{m+1-(k+1)}$, where $P_{22}=\left(\begin{array}{c}P_{22}^{\prime} \\ z_{1} \\ z_{2}\end{array}\right)_{1}^{k-1} \begin{gathered}1 \\ 1\end{gathered}$. Then $P_{1}=\left(\begin{array}{cc}P_{11} & P_{12} \\ 0 & 2_{22}^{\prime} \\ 0 & z_{1}\end{array}\right)$ and $P_{2}=\left(\begin{array}{cc}P_{11} & P_{12} \\ 0 & P_{22}^{\prime} \\ 0 & z_{2}\end{array}\right) \begin{gathered}m+1-(k+1) \\ k-1 \\ 1\end{gathered}$ are subspace of type $(m, s, k)$, such that $P=P_{1}+P_{2}$.
Lemma 2. Let $2 v+l>0,0 \leq k \leq l, 2 s \leq m-k \leq v+s$ and $m \neq 2 v+l$. For any subspace $P$ of type $(m+1, s+1, k)$, there are two subspaces $P_{1}$ and $P_{2}$ of type $(m, s, k)$ such that $P=P_{1}+P_{2}$.

Proof. Assume that $P K_{v} P^{T}=\left(\begin{array}{c}P_{0} \\ x_{1} \\ y_{1}\end{array}\right) K_{v}\left(\begin{array}{c}P_{0} \\ x_{1} \\ y_{1}\end{array}\right)^{T}=$

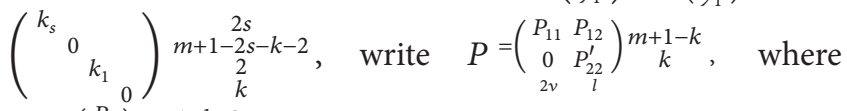
$P_{11}=\left(\begin{array}{c}P_{0} \\ x_{1} \\ y_{1}\end{array}\right)^{m+1-k-2} \begin{gathered}1 \\ 1\end{gathered}, x_{1}$ and $y_{1}$ are the $(m+1-(k+1))$-th and $(m-k+1)$-th row vectors of $P_{11}$, respectively.

$P_{12}=\left(\begin{array}{c}P_{0}^{\prime} \\ x_{1}^{\prime} \\ y_{1}^{\prime}\end{array}\right) \begin{gathered}m+1-k-2 \\ 1\end{gathered}, x_{1}^{\prime}$ and $y_{1}^{\prime}$ are the $(m+1-(k+1))$-th and $(m-k+1)$-th row vectors of $P_{12}$, respectively.

Then

$$
\begin{aligned}
& P_{1}=\left(\begin{array}{cc}
P_{0} & P_{0}^{\prime} \\
x_{1} & x_{1}^{\prime} \\
0 & P_{22}
\end{array}\right) \\
& \begin{array}{cc}
m+1-k-2 \\
1 \\
k,
\end{array} \\
& P_{2}=\left(\begin{array}{cc}
P_{0} & P_{0}^{\prime} \\
y_{1} & y_{1}^{\prime} \\
0 & P_{22}
\end{array}\right) \\
& m+1-k-2 \\
& 1 \\
& k
\end{aligned}
$$

are subspace of type $(m, s, k)$, such that $P=P_{1}+P_{2}$.

Theorem 3. Let $2 v+l>0$, assume that $(m, s, k),\left(m_{1}, s_{1}, k_{1}\right)$ satisfy $0 \leq k \leq l, 2 s \leq m-k \leq v+s, 0 \leq k_{1} \leq l, 2 s \leq m_{1}-k_{1} \leq$ $v+s$. For any subspace $P$ of type $(m, s, k)$, there are subspace $P_{1}, P_{2}, \ldots, P_{i}$ of type $\left(m_{1}, s_{1}, k_{1}\right)$ such that $P=P_{1}+P_{2}+\cdots+P_{i}$ if and only if

$$
\begin{gathered}
\text { (i) } s=s_{1}=v, \quad 0 \leq k_{1} \leq k \leq l, \\
\text { (ii) } s<v, \quad k_{1}=k=l, \\
m-m_{1} \geq s-s_{1} \geq 0, \\
\text { (iii) } s<v, \quad 0 \leq k_{1} \leq k \leq l, \\
(m-k)-\left(m_{1}-k_{1}\right) \geq s-s_{1} \geq 0 .
\end{gathered}
$$

Proof. Suppose that $(m, s, k)$ and $\left(m_{1}, s_{1}, k_{1}\right)$ satisfy condition (7). Let $k-k_{1}=h(h \geq 0)$, since $s=s_{1}=v, m=2 v+$ $k, m_{1}=2 v+k_{1}, m-m_{1}=k-k_{1}=h$, By Lemma 1 the desired result follows. Suppose $(m, s, k)$ and $\left(m_{1}, s_{1}, k_{1}\right)$ satisfy condition (8). Let $s-s_{1}=t, m-m_{1}=t+t^{\prime}\left(t, t^{\prime} \geq 0\right)$. By Lemma 1 , any subspace of type $(m, s, l)$ is the sum some subspaces of type $(m-t, s, l)$. By Lemma 2 , any subspace of type $(m-t, s, l)$ is the sum some subspaces of type $\left(m_{1}, s_{1}, l\right)$. Hence the desired result follows.

Suppose $(m, s, k)$ and $\left(m_{1}, s_{1}, k_{1}\right)$ satisfy condition (9). Let $s-s_{1}=t, k-k_{1}=h, m-m_{1}=t+h+$ $t^{\prime}\left(t, t^{\prime}, h \geq 0\right)$, By Lemma 1 , we have any subspace of type $(m, s, k)$ is the sum some subspace of type $\left(m-t^{\prime}, s, k\right)$. By Lemma 2, we have any subspace of type $\left(m-t^{\prime}, s, k\right)$ is the sum some subspace of type $\left(m-t^{\prime}-t, s_{1}, k\right)$. By Lemma 1 , we have any subspace of type $\left(m-t^{\prime}-t, s_{1}, k\right)$ is the sum some subspace of type $\left(m_{1}, s_{1}, k_{1}\right)$. Hence the desired result follows.

Conversely, If $s_{1}=v$, then $s=v, k_{1} \leq l$. Let $Q \in$ $M(m, v, k ; 2 v+l, v)$, there exists $P \in M\left(m_{1}, v, k_{1} ; 2 v+l, v\right)$, 
such that $P \subset Q$, hence $P \cap E \subset Q \cap E, k_{1}=\operatorname{dim}(P \cap E) \leq$ $\operatorname{dim}(Q \cap E)=k$. Therefore, $s=s_{1}=v, 0 \leq k_{1} \leq k \leq l$, condition (7) hold.

If $s_{1}<v$, let $P \in M\left(m_{1}, v, k_{1} ; 2 v+l, v\right), Q \in M(m, v, k$ : $2 v+l, v)$, such that $P \subset Q$, then $P \cap E \subset Q \cap E$, and $k_{1}=$ $\operatorname{dim}(P \cap E) \leq \operatorname{dim}(Q \cap E)=k$. If $k_{1}=l$, then $k=l$ and $m_{1} \geq l$, Assume $Q=\left(\begin{array}{cc}Q_{11} & Q_{12} \\ 0 & Q_{22}\end{array}\right)^{m-1}$ where rank $Q_{11}=m-l$, rank $2 v \quad l$

$Q_{22}=l$ and $Q_{11} K Q^{t}=M(m-l, s)$. Since $P \cap E \subset Q \cap E$, there exists a $l \times l$ matrix $P_{22}$ with $\operatorname{rank} P_{22}=l$, such that $P_{22} Q_{22}$ is a matrix representation of subspace $P \cap E$. Thus we can assume $P=\left(\begin{array}{cc}P_{11} & P_{12} \\ 0 & P_{22}\end{array}\right) \begin{gathered}m_{1}-1 \\ l\end{gathered}$, where $P_{11}$ is a matrix with rank $m_{1}-l$. $2 v l$

Because $P$ is a subspaces of type $\left(m_{1}, s_{1}, l\right)$, we can assume $P_{11} M(m-l, s) P_{11}^{t}=M\left(m_{1}-l, s\right)$. Then $Q_{11}$ can be considered as a subspace of type $\left(m_{1}-l, s_{1}\right)$ in singular symplectic space $F_{q}^{(2 s+(m-2 s-l))}$. Hence $m_{1}-l-s-s_{1} \leq m-2 s-l$, condition (8) hold. Similarly we also can prove condition (9) hold.

Theorem 4. Let $2 v+l>0$, assume that $(m, s, k)$ and $\left(m_{2}, s_{2}, k_{2}\right)$ satisfy $0 \leq k \leq l, 2 s \leq m-k \leq v+s, 0 \leq k_{2} \leq l$, $2 s_{2} \leq m_{2}-k_{2} \leq v+s$. For any subspace $P$ of type $(m, s, k)$, there are subspace $P_{1}, P_{2}, \ldots, P_{j}$ of type $\left(m_{2}, s_{2}, k_{2}\right)$ such that $P=P_{1} \cap P_{2} \cap \cdots \cap P_{j}$ if and only if

(i) $s=s_{2}=v, 0 \leq k_{2} \leq k \leq l$,

(ii) $s<v, k_{2}=k=l$ and $m_{2}-m \geq s_{2}-s \geq 0$,

(iii) $s<v, k \leq k_{2} \leq l$ and $\left(m_{2}-k_{2}\right)-(m-k) \geq s_{2}-s \geq 0$.

Proof. By [3], it is directed.

Theorem 5. For $1 \leq m_{1} \leq m_{2} \leq 2 v+l, L\left(m_{1}, s_{1}, k_{1}\right.$; $\left.m_{2}, s_{2}, k_{2} ; 2 v+l, v\right)$ consist of $\{0\}, F_{q}^{(2 v+l)}$ and all subspaces of type $(m, s, k)$ in $F_{q}^{(2 v+l)}$ such that

(i) $s=s_{1}=s_{2}=v, 0 \leq k_{1} \leq k_{2} \leq k \leq l$,

(ii) $s<v, k_{1}=k=k_{2}=l$ and $m-m_{1} \geq s-s_{1} \geq 0$, $m_{2}-m \geq s_{2}-s \geq 0$,

(iii) $s<v, k_{1} \leq k \leq k_{2} \leq l$ and $\left(m_{2}-k_{2}\right)-(m-k) \geq$ $s_{2}-s \geq 0,(m-k)-\left(m_{1}-k_{1}\right) \geq s-s_{1} \geq 0$.

Proof. By Theorems 3 and 4, it is directed.

\section{The Geometricity of Lattices}

$$
L_{O}\left(m_{1}, s_{1}, k_{1} ; m_{2}, s_{2}, k_{2} ; 2 v+l, v\right) \text { and }
$$$$
L_{R}\left(m_{1}, s_{1}, k_{1} ; m_{2}, s_{2}, k_{2} ; 2 v+l, v\right)
$$

Lemma 6 (see [3]). If $0<k<l$, then

(i) $L_{R}(2 v+k, v, k ; 2 v+l, v) \simeq L_{R}(k, l), L_{O}(2 v+k, v, k ; 2 v+$ $l, v) \simeq L_{O}(k, l)$,

(ii) $L_{R}(k, 0, k ; 2 v+l, v) \simeq L_{R}(k, l), L_{O}(k, 0, k ; 2 v+l, v) \simeq$ $L_{O}(k, l)$.

Lemma 7 (see [3]). If $0 \leq s<v$ and $2 s \leq m-l \leq v+s$, then

(i) $L_{R}(m, s, l ; 2 v+l, v) \simeq L_{R}(m-l, s ; 2 v), L_{O}(m, s, l ; 2 v+$ $l, v) \simeq L_{O}(m-l, s ; 2 v)$, (ii) $L_{R}(m, s, 0 ; 2 v+l, v) \simeq L_{R}(m, s ; 2 v), L_{O}(m, s, 0 ; 2 v+$ $l, v) \simeq L_{\mathrm{O}}(m, s ; 2 v)$.

Theorem 8. Let $2 v+l>0$. Assume that $\left(m_{1}, s_{1}, k_{1}\right)$, $\left(m_{2}, s_{2}, k_{2}\right)$ satisfies $0 \leq k_{1} \leq l, 2 s_{1} \leq m_{1}-k_{1} \leq v+s_{1}$, $0 \leq k_{2} \leq l, 2 s_{2} \leq m_{2}-k_{2} \leq v+s_{2}$ and $1 \leq m_{1} \leq m_{2}<2 v+l$. Then

(i) $L_{O}(k+1,0, k ; 2 v-1+k, v-1, k ; 2 v+l, v)$ is a finite geometric lattice if and only if $k=0, l$,

(ii) $L_{O}(k, 0, k ; 2 v+k, v, k ; 2 v+l, v)$ is a finite geometric lattice if and only if $k=1, l-1$,

(iii) $L_{O}\left(m_{1}, s_{1}, k_{1} ; m_{2}, s_{2}, k_{2} ; 2 v+l, v\right)$ is not a geometric lattice when $2 \leq m_{1}-k_{1} \leq m_{2}-k_{2} \leq 2 v-2$.

Proof. For any $X \in L_{O}\left(m_{1}, s_{1}, k_{1} ; m_{2}, s_{2}, k_{2} ; 2 v+l, v\right)$, define

$$
r_{O}(X)= \begin{cases}0, & \text { if } X=0 \\ m_{2}-m_{1}+2, & \text { if } X=F_{q}^{(2 v+l)} \\ \operatorname{dim} X-m_{1}+1, & \text { otherwise. }\end{cases}
$$

Then $r_{O}$ is the rank function of $L_{O}\left(m_{1}, s_{1}, k_{1} ; m_{2}, s_{2}, k_{2} ; 2 v+\right.$ $l, v)$.

(i) For lattice $L_{O}(k+1,0, k ; 2 v-1+k, v-1, k$; $2 v+l, v)$. If $k=0$, by Lemma $7, L_{O}(1,0,0 ; 2 v-l, v-1,0 ; 2 v+l, v) \simeq$ $L_{O}(1,0,2 v-1, v-1 ; 2 v)$, by [12], $L_{O}(k+1,0, k ; 2 v-1+k, v-$ $1, k ; 2 v+l, v)$ is a finite geometric lattice.

If $k=l$, by Lemma $7, L_{O}(l+1,0, l ; 2 v-l+l, v-1, l ; 2 v+$ $l, v) \simeq L_{O}(1,0,2 v-1, v-1 ; 2 v)$, by [12], $L_{O}(k+1,0, k ; 2 v-$ $1+k, v-1, k ; 2 v+l, v)$ is a finite geometric lattice.

If $0<k<l$. Let $U=\left\langle e_{1}, e_{2 v+1}, \ldots, e_{2 v+k}\right\rangle$ and $W=$ $\left\langle e_{v+1}, e_{2 v+2}, \ldots, e_{2 v+k+1}\right\rangle$, then $U$ and $W$ both are of type $(k+$ $1,0, k),\langle U, W\rangle$ is of type $(k+3,1, k+1),\langle U, W\rangle \in L_{O}(k+$ $1,0, k ; 2 v-1+k, v-1, k ; 2 v+l, v), U \cap W$ is of type $(k-1,0, k-1)$, hence $r_{O}(U \wedge W)=0, r_{O}(U \vee W)=3$, $r_{O}(U)=r_{O}(W)=1$. We have

$$
r_{O}(U \wedge W)+r_{O}(U \vee W)=3>r_{O}(U)+r_{O}(W)=2 .
$$

That is $L_{O}(k+1,0, k ; 2 v-1+k, v-1, k ; 2 v+l, v)$ is not a geometric lattice when $0<k<l$.

(ii) For lattice $L_{O}(k, 0, k ; 2 v+k, v, k ; 2 v+l, v)$, by Lemma $6 L_{O}(k, 0, k ; 2 v+k, v, k ; 2 v+l, v) \simeq L_{O}(k, l)$.

If $k=1, l-1, L_{O}(1, l)$ and $L_{O}(l-1, l)$ is a geometric lattice.

If $2 \leq k \leq l-2$, let $v_{1}, v_{2}, \ldots, v_{l}$ be a basis of $F_{q}^{(l)}$. Since $2 \leq k \leq l-2$, we can take $U=\left\langle v_{1}, v_{2}, \ldots, v_{k}\right\rangle$, $W=\left\langle v_{3}, v_{4}, \ldots, v_{k+2}\right\rangle \in L_{O}(k, l)$. Hence $U \wedge W=\{0\}$, $U \vee W=\langle U, W\rangle, r_{O}(U \wedge W)=0, r_{O}(U \vee W)=3$, $r_{O}(U)=r_{O}(W)=1$. We have

$$
r_{O}(U \wedge W)+r_{O}(U \vee W)=3>r_{O}(U)+r_{O}(W)=2
$$

That is $L_{\mathrm{O}}(k, 0, k ; 2 v+k, v, k ; 2 v+l, v)$ is not a geometric lattice when $2 \leq k \leq l-2$.

(iii) For lattice $L_{\mathrm{O}}\left(m_{1}, s_{1}, k_{1} ; m_{2}, s_{2}, k_{2} ; 2 v+l, v\right)$, when $2 \leq$ $m_{1}-k_{1} \leq m_{2}-k_{2} \leq 2 v-2$. 
Case (a). $k_{1} \leq k_{2} \leq l$.

$$
\begin{aligned}
& U=\left(\begin{array}{ccccccccc}
I^{\left(s_{2}-1\right)} & 0 & 0 & 0 & 0 & 0 & 0 & 0 & 0 \\
0 & 0 & 0 & 0 & I^{\left(s_{2}\right)} & 0 & 0 & 0 & 0 \\
0 & 0 & I^{\left(m_{2}-k_{2}-2 s_{2}\right)} & 0 & 0 & 0 & 0 & 0 & 0 \\
0 & 0 & 0 & 0 & 0 & 0 & 0 & I^{\left(k_{2}\right)} & 0
\end{array}\right), \\
& \left.W=\begin{array}{cccccccccc}
0 & 0 & 0 & 1 & 0 & 0 & 0 & 0 & 0 & 0 \\
0 & 0 & 0 & 0 & 0 & 0 & 0 & 0 & I^{\left(k_{2}\right)} & 0
\end{array}\right) .
\end{aligned}
$$

Then $U$ is a of type $\left(m_{2}-1, s_{2}-1, k_{2}\right), W$ is a of type $\left(k_{2}+1,0, k_{2}\right),\langle U, W\rangle$ is a of type $\left(m_{2}, s_{2}-1, k_{2}\right), r_{O}(U \vee W)=$ $r_{O}\left(F_{q}^{(2 v+l)}\right)=m_{2}-m_{1}+2, r_{O}(U \wedge W)=k_{2}-m_{1}+1$, $r_{O}(U)=m_{2}-1-m_{1}+1=m_{2}-m_{1}, r_{O}(W)=k_{2}+1-m_{1}+1=$ $k_{2}-m_{1}+2, r_{O}(U \vee W)+r_{O}(U \wedge W)=m_{2}-2 m_{1}+k_{2}+3$, $r_{O}(U)+r_{O}(W)=m_{2}-2 m_{1}+k_{2}+2$. We have

$$
r_{O}(U \vee W)+r_{O}(U \wedge W)>r_{O}(U)+r_{O}(W) .
$$

Hence, $L_{O}\left(m_{1}, s_{1}, k_{1} ; m_{2}, s_{2}, k_{2} ; 2 v+l, v\right)$ is not a geometric lattice when $\left(\mathrm{a}_{1}\right)$.

$\left(\mathrm{a}_{2}\right) s_{2}>0, m_{2}-k_{2}=v+s_{2}$ or $s_{2}=0$.

When $s_{2}=0$, we have $m_{2}-k_{2}-2 s_{2} \geq 1$, when $s_{2}>0, m_{2}-k_{2}=$ $v+s_{2}$, we have $v-2 \geq s_{2}, m_{2}-k_{2}-2 s_{2}-1=v-s_{2}-1 \geq 1$.

Let

$$
\begin{aligned}
& U=\left(\begin{array}{cccccccccc}
I^{\left(s_{2}\right)} & 0 & 0 & 0 & 0 & 0 & 0 & 0 & 0 & 0 \\
0 & 0 & 0 & 0 & I^{\left(s_{2}\right)} & 0 & 0 & 0 & 0 & 0 \\
0 & I^{\left(m_{2}-k_{2}-2 s_{2}\right)} & 0 & 0 & 0 & 0 & 0 & 0 & 0 & 0 \\
0 & 0 & 0 & 0 & 0 & 0 & 0 & 0 & I^{\left(k_{2}\right)} & 0
\end{array}\right), \\
& W=\left(\begin{array}{cccccccc}
0 & 0 & 0 & 1 & 0 & 0 & 0 & 0 \\
0 & 0 & 0 & 0 & 0 & 0 & I^{\left(k_{2}\right)} & 0
\end{array}\right) .
\end{aligned}
$$

Then $U$ is a of type $\left(m_{2}-1, s_{2}, k_{2}\right), W$ is a of type $\left(k_{2}+\right.$ $\left.1,0, k_{2}\right),\langle U, W\rangle$ is a of type $\left(m_{2}, s_{2}+1, k_{2}\right)$, clearly, we have

$$
r_{O}(U \vee W)+r_{O}(U \wedge W)>r_{O}(U)+r_{O}(W) .
$$

Hence, $L_{O}\left(m_{1}, s_{1}, k_{1} ; m_{2}, s_{2}, k_{2} ; 2 v+l, v\right)$ is not a geometric lattice when $\left(\mathrm{a}_{2}\right)$.

Case (b). $k_{1}=k_{2}=l$.

In this case $L_{O}\left(m_{1}, s_{1}, l ; m_{2}, s_{2}, l ; 2 v+l, v\right) \simeq L_{O}\left(m_{1}-\right.$ $\left.l, s_{1} ; m_{2}-l, s_{2} ; 2 v\right), L_{O}\left(m_{1}, s_{1}, l ; m_{2}, s_{2}, l ; 2 v+l, v\right)$ is not a geometric lattice.

Hence $L_{O}\left(m_{1}, s_{1}, k_{1} ; m_{2}, s_{2}, k_{2} ; 2 v+l, v\right)$ is not a geometric lattice when $2 \leq m_{1}-k_{1} \leq m_{2}-k_{2} \leq 2 v-2$.

Theorem 9. Let $2 v+l>0$. Assume that $\left(m_{1}, s_{1}, k_{1}\right)$, $\left(m_{2}, s_{2}, k_{2}\right)$ satisfies $0 \leq k_{1} \leq l, 2 s_{1} \leq m_{1}-k_{1} \leq v+s_{1}$, $0 \leq k_{2} \leq l, 2 s_{2} \leq m_{2}-k_{2} \leq v+s_{2}$ and $1 \leq m_{1} \leq m_{2}<2 v+l$. Then

(i) $L_{R}(k+1,0, k ; 2 v-1+k, v-1, k ; 2 v+l, v)$ is a finite geometric lattice if and only if $k=0, l$,

(ii) $L_{R}(k, 0, k ; 2 v+k, v, k ; 2 v+l, v)$ is a finite geometric lattice if and only if $k=1, l-1$, (iii) $L_{R}\left(m_{1}, s_{1}, k_{1} ; m_{2}, s_{2}, k_{2} ; 2 v+l, v\right)$ is not a geometric lattice when $2 \leq m_{1}-k_{1} \leq m_{2}-k_{2} \leq 2 v-2$.

Proof. (i) For $X \in L_{R}\left(m_{1}, s_{1}, k_{1} ; m_{2}, s_{2}, k_{2} ; 2 v+l, v\right)$, define

$$
r_{R(X)}= \begin{cases}0, & \text { if } X=F_{q}^{((2 v+l))}, \\ m_{2}-m_{1}+2, & \text { if } X=0, \\ m_{2}+1-\operatorname{dim} X, & \text { otherwise. }\end{cases}
$$

For lattice $L_{R}(k+1,0, k ; 2 v-1+k, v-1, k ; 2 v+l, v)$.

If $k=0$, by Lemma $7, L_{R}(1,0,0 ; 2 v-l, v-1,0 ; 2 v+l, v) \simeq$ $L_{R}(1,0,2 v-1, v-1 ; 2 v)$, by [12], $L_{R}(k+1,0, k ; 2 v-1+k, v-$ $1, k ; 2 v+l, v)$ is a finite geometric lattice.

If $k=l$, by Lemma $7, L_{R}(l+1,0, l ; 2 v-l+l, v-1, l ; 2 v+$ $l, v) \simeq L_{R}(1,0,2 v-1, v-1 ; 2 v)$, by [12], $L_{R}(k+1,0, k ; 2 v-$ $1+k, v-1, k ; 2 v+l, v)$ is a finite geometric lattice.

If $0<k<l$, let $U=\left\langle e_{1}, \ldots, e_{v-1}, e_{v+1}, \ldots\right.$, $\left.e_{2 v}, e_{2 v+1}, \ldots, e_{2 v+k}\right\rangle, W=\left\langle e_{2}, \ldots, e_{v}, e_{v+2}, \ldots, e_{2 v+1}, e_{2 v+2}\right.$, $\left.\ldots, e_{2 v+k}\right\rangle$. Then $U, W$ both are of type $(2 v-1+k, v-1, k)$, $U \wedge W=\langle U, W\rangle$ is of type $(2 v+1+k, v, k+1), U \vee W=U \cap W$ is of type $(2 v-3+k, v, k)$. We have

$$
r(U \wedge W)+r(U \vee W)=3>r(U)+r(W)=2 .
$$


Hence, $L_{R}(k+1,0, k ; 2 v-1+k, v-1, k ; 2 v+l, v)$ is not a geometric lattice when $0<k<l$. (ii) if $k=0, l-1$, by Lemma $7 L_{R}(k, 0, k ; 2 v+k, v, k ; 2 v+$ $l, v) \simeq L_{R}(k, l)$ when $k=1, l-1, L_{R}(k, 0, k ; 2 v+k, v, k ; 2 v+l, v)$ is a geometric lattice.

If $2 \leq k \leq l-2$, by [7], $L_{R}(k, l)$ is not a geometric lattice.

(iii) Case (a). if $m_{1}-k_{1}<v+s_{1}$, then $v+s_{1}-m_{1}+k_{1}-1 \geq 0$. Let

$$
\begin{aligned}
& U=\left(\begin{array}{cccccccccc}
I^{\left(s_{1}\right)} & 0 & 0 & 0 & 0 & 0 & 0 & 0 & 0 & 0 \\
0 & 0 & 0 & 0 & I^{\left(s_{1}\right)} & 0 & 0 & 0 & 0 & 0 \\
0 & 1 & 0 & 0 & 0 & 0 & 0 & 0 & 0 & 0 \\
0 & 0 & I^{\left(m_{1}-k_{1}-2 s_{1}\right)} & 0 & 0 & 0 & 0 & 0 & 0 & 0 \\
0 & 0 & 0 & 0 & 0 & 0 & 0 & 0 & I^{\left(k_{1}\right)} & 0
\end{array}\right) \\
& s_{1} \quad 1 \quad m_{1}-k_{1}-2 s_{1} \quad v+s_{1}-m_{1}+k_{1}-1 \quad s_{1} \quad 1 \quad m_{1}-k_{1}-2 s_{1} \quad v+s_{1}-m_{1}+k_{1} \quad k_{1} \quad l-k_{1} \\
& W=\left(\begin{array}{cccccccccc}
0 & I^{\left(s_{1}\right)} & 0 & 0 & 0 & 0 & 0 & 0 & 0 & 0 \\
0 & 0 & 0 & 0 & 0 & I^{\left(s_{1}\right)} & 0 & 0 & 0 & 0 \\
0 & 0 & 0 & 0 & 1 & 0 & 0 & 0 & 0 & 0 \\
0 & 0 & I^{\left(m_{1}-k_{1}-2 s_{1}\right)} & 0 & 0 & 0 & 0 & 0 & 0 & 0 \\
0 & 0 & 0 & 0 & 0 & 0 & 0 & 0 & I^{\left(k_{1}\right)} & 0
\end{array}\right) .
\end{aligned}
$$

Then $U, W$ are of type $\left(m_{1}+1, s_{1}, k_{1}\right), U \cap W$ is of type $\left(m_{1}, s_{1}-1, k_{1}\right),\langle U, W\rangle$ is of type $\left(m_{1}+1, s_{1}, k_{1}\right)$, $\langle U, W\rangle \in L_{R}\left(m_{1}, s_{1}, k_{1} ; m_{2}, s_{2}, k_{2} ; 2 v+l, v\right), U \cap W \notin$ $L_{R}\left(m_{1}, s_{1}, k_{1} ; m_{2}, s_{2}, k_{2} ; 2 v+l, v\right)$.

So $r_{R}(U \vee W)=m_{2}-m_{1}+2, r_{R}(U \wedge W)=m_{2}-m_{1}-1$, $r_{R}(U)=m_{2}+1-\left(m_{1}+1\right)=m_{2}-m_{1}=r_{R}(W)$. We have

$$
\begin{aligned}
r_{R}(U & \wedge W)+r_{R}(U \vee W) \\
& =2 m_{2}-2 m_{1}+1
\end{aligned}
$$

$$
\begin{aligned}
& U=\left(\begin{array}{ccccc}
I^{\left(s_{1}\right)} & 0 & 0 & 0 & 0 \\
0 & 0 & 0 & 0 & 0 \\
0 & 1 & 0 & 0 & 0 \\
0 & 0 & 1 & 0 & 0 \\
0 & 0 & 0 & I^{\left(m_{1}-k_{1}-2 s_{1}\right)} & 0 \\
0 & 0 & 0 & 0 & 0 \\
0 & 0 & 0 & 0 & 0 \\
s_{1} & 1 & 1 & m_{1}-k_{1}-2 s_{1} & v+s_{1}-m_{1}+k_{1}
\end{array}\right. \\
& W=\left(\begin{array}{ccccc}
I^{\left(s_{1}\right)} & 0 & 0 & 0 & 0 \\
0 & 0 & 0 & 0 & 0 \\
0 & 0 & 0 & 0 & 0 \\
0 & 0 & 1 & 0 & 0 \\
0 & 0 & 0 & I^{\left(m_{1}-k_{1}-2 s_{1}\right)} & 0 \\
0 & 0 & 0 & 0 & 0 \\
0 & 0 & 0 & 0 & 0 \\
s_{1} & 1 & 1 & m_{1}-k_{1}-2 s_{1} & v+s_{1}-m_{1}+k_{1}
\end{array}\right.
\end{aligned}
$$

Then $U, W$ are of type $\left(m_{1}+1, s_{1}+1, k_{1}\right), U \cap W$ is of type $\left(m_{1}, s_{1}+1, k_{1}\right)$, and $\langle U, W\rangle$ is of type $\left(m_{1}+2, s_{1}+2\right.$, $\left.k_{1}\right)$.

$$
\begin{aligned}
& >r_{R}(U)+r_{R}(W) \\
& =2 m_{2}-2 m_{1} .
\end{aligned}
$$

Hence $L_{R}\left(m_{1}, s_{1}, k_{1} ; m_{2}, s_{2}, k_{2} ; 2 v+l, v\right)$ is not a geometric lattice when (a).

Case (b). $m_{1}-k_{1}=v+s_{1}$, from $m_{1}-k_{1}=v+s_{1}$, and $2 \leq m_{1}-k_{1} \leq 2 v-2$, we have $v-2 \geq s_{1}, m_{1}-k_{1}-2 s_{1}-2=$ $v-s_{1}-2 \geq 0$.

Let

$\begin{array}{ccccccc}0 & 0 & 0 & 0 & 0 & 0 & 0 \\ I^{\left(s_{1}\right)} & 0 & 0 & 0 & 0 & 0 & 0 \\ 0 & 0 & 0 & 0 & 0 & 0 & 0 \\ 0 & 0 & 0 & 0 & 0 & 0 & 0 \\ 0 & 0 & 0 & 0 & 0 & 0 & 0 \\ 0 & 0 & 1 & 0 & 0 & 0 & 0 \\ 0 & 0 & 0 & 0 & 0 & I^{\left(k_{1}\right)} & 0 \\ s_{1} & 1 & 1 & m_{1}-k_{1}-2 s_{1} & v+s_{1}-m_{1}+k_{1} & k_{1} & l-k_{1}\end{array}$

$\left.\begin{array}{ccccccc}0 & 0 & 0 & 0 & 0 & 0 & 0 \\ I^{\left(s_{1}\right)} & 0 & 0 & 0 & 0 & 0 & 0 \\ 0 & 1 & 0 & 0 & 0 & 0 & 0 \\ 0 & 0 & 0 & 0 & 0 & 0 & 0 \\ 0 & 0 & 0 & 0 & 0 & 0 & 0 \\ 0 & 0 & 1 & 0 & 0 & 0 & 0 \\ 0 & 0 & 0 & 0 & 0 & I^{\left(k_{1}\right)} & 0\end{array}\right)$. 
Hence $L_{R}\left(m_{1}, s_{1}, k_{1} ; m_{2}, s_{2}, k_{2} ; 2 v+l, v\right)$ is not a geometric lattice when (b).

Hence $L_{R}\left(m_{1}, s_{1}, k_{1} ; m_{2}, s_{2}, k_{2} ; 2 v+l, v\right)$ is not a geometric lattice when $2 \leq m_{1}-k_{1} \leq m_{2}-k_{2} \leq 2 v-2$.

\section{Characteristic Polynomial of Lattice}

$$
L_{R}\left(m_{1}, s_{1}, k_{1} ; m_{2}, s_{2}, k_{2} ; 2 v+l, v\right)
$$

In this section we compute the characteristic polynomial of the lattice $L_{R}\left(m_{1}, s_{1}, k_{1} ; m_{2}, s_{2}, k_{2} ; 2 v+l, v\right)$.

Theorem 10. Let $2 v+l>0$, Assume that $(m, s, k)$ satisfies $0 \leq k \leq l, 2 s \leq m-k \leq v+s$ and $0<m<2 v+l$. Then

$$
\begin{aligned}
\chi & \left(L_{R}\left(m_{1}, s_{1}, k_{1} ; m_{2}, s_{2}, k_{2} ; 2 v+l, v\right), t\right) \\
= & t^{m_{2}-m_{1}+2} \\
& -\sum_{k=k_{1}}^{k} \sum_{s=s_{1}}^{s_{2}} \sum_{m=m_{2}-\left(k_{2}-k\right)+\left(s_{2}-s\right)+1}^{m_{1}-\left(k-k_{1}\right)+\left(s-s_{1}\right)} N(m, s, k ; 2 v+l, v) g_{m}(t) \\
& +\sum_{k=0}^{k_{1}} \sum_{s=0}^{s_{1}-1} \sum_{m=2 s+k}^{v+s+k} N(m, s, k ; 2 v+l, v) g_{m}(t) \\
& +\sum_{k=k_{2}}^{l} \sum_{s=s_{2}+1}^{v} \sum_{m=2 s+k}^{v+s+k} N(m, s, k ; 2 v+l, v) g_{m}(t),
\end{aligned}
$$

where $g_{m}(t)=\prod_{i=0}^{m-1}\left(t-q^{i}\right)$.

Proof. Define

$$
r_{R}(X)= \begin{cases}0, & \text { if } X=F_{q}^{(2 v+l),} \\ m_{2}-m_{1}+2, & \text { if } X=\{0\}, \\ m_{2}+1-\operatorname{dim}(X), & \text { otherwise. }\end{cases}
$$

Then $r_{R}$ is the rank function on $L_{R}\left(m_{1}, s_{1}, k_{1} ; m_{2}, s_{2}\right.$, $\left.k_{2} ; 2 v+l, v\right)$. Let $V=F_{q}^{(2 v+l)}, L_{O}=L_{R}(2 v+l, v), L=$ $L_{R}\left(m_{1}, s_{1}, k_{1} ; m_{2}, s_{2}, k_{2} ; 2 v+l, v\right)$ we have

$$
\chi\left(L_{O}, t\right)=\prod_{i=0}^{2 v+l-1}\left(t-q^{i}\right) .
$$

For any $P \in L$, define

$$
\begin{gathered}
L^{P}=\{Q \in L \mid Q \subset P\}=\{Q \in L \mid Q \geq P\}, \\
L_{O}^{P}=\left\{Q \in L_{O} \mid Q \subset P\right\}=\left\{Q \in L_{O} \mid Q \geq P\right\} .
\end{gathered}
$$

Clearly, $L^{V}=L, L^{P}=L_{O}^{P}$, when $P \neq\{0\}, P \neq V$

$$
\begin{aligned}
\chi\left(L^{V}, t\right) & =\chi(L, t)=\sum_{P \in L} \mu(0, P) t^{r(1)-r(P)} \\
& =\sum_{P \in L} \mu(0, P) t^{r(0)-r(P)} .
\end{aligned}
$$

By inversion to Möbius we have

$$
\chi(L, t)=\chi\left(L^{V}, t\right)=t^{m_{2}-m_{1}+2}-\sum_{P \in L \backslash\{V\}} \chi\left(L^{P}, t\right),
$$

by Theorem 5 we have

$$
\begin{aligned}
\chi( & \left.L_{R}\left(m_{1}, s_{1}, k_{1} ; m_{2}, s_{2}, k_{2} ; 2 v+l, v\right), t\right) \\
= & t^{m_{2}-m_{1}+2} \\
& -\sum_{k=k_{1}}^{k_{2}} \sum_{s=s_{1}}^{s_{2}} \sum_{m=m_{2}-\left(k_{2}-k\right)+\left(s_{2}-s\right)+1}^{m_{1}-\left(k-k_{1}\right)+\left(s-s_{1}\right)} N(m, s, k ; 2 v+l, v) g_{m}(t) \\
& +\sum_{k=0}^{k_{1}} \sum_{s=0}^{s_{1}-1} \sum_{m=2 s+k}^{v+s+k} N(m, s, k ; 2 v+l, v) g_{m}(t) \\
& +\sum_{k=k_{2}}^{l} \sum_{s=s_{2}+1}^{v} \sum_{m=2 s+k}^{v+s+k} N(m, s, k ; 2 v+l, v) g_{m}(t) .
\end{aligned}
$$

\section{Acknowledgments}

This work is supported by the National Natural Science Foundation of China under Grant no. 61179026 and Supported by the Fundamental Research Funds for the Central Universities (ZXH2012 K003).

\section{References}

[1] M. Aigner, Combinatorial Theory, vol. 234, Springer, Berlin, Germany, 1979.

[2] Z. X. Wan, Geometry of Classical Groups Over Finite Fields, Studentlitteratur, Lund, Sweden, 1993.

[3] Y. Gao and H. You, "Lattices generated by orbits of subspaces under finite singular classical groups and its characteristic polynomials," Communications in Algebra, vol. 31, no. 6, pp. 2927-2950, 2003.

[4] Y. J. Huo, Y. Liu, and Z. X. Wan, "Lattices generated by transitive sets of subspaces under finite classical groups. I," Communications in Algebra, vol. 20, no. 4, pp. 1123-1144, 1992.

[5] Y. J. Huo, Y. Liu, and Z. X. Wan, "Lattices generated by transitive sets of subspaces under finite classical groups. II. The orthogonal case of odd characteristic," Communications in Algebra, vol. 20, no. 9, pp. 2685-2727, 1992.

[6] Y. J. Huo, Y. Liu, and Z. X. Wan, "Lattices generated by transitive sets of subspaces under finite classical groups. III. The orthogonal case of even characteristic," Communications in Algebra, vol. 21, no. 7, pp. 2351-2393, 1993.

[7] Y. J. Huo and Z. X. Wan, "On the geometricity of lattices generated by orbits of subspaces under finite classical groups," Journal of Algebra, vol. 243, no. 1, pp. 339-359, 2001.

[8] P. Orlik and L. Solomon, "Arrangements in unitary and orthogonal geometry over finite fields," Journal of Combinatorial Theory, Series A, vol. 38, no. 2, pp. 217-229, 1985.

[9] K. Wang and Y.-q. Feng, "Lattices generated by orbits of flats under finite affine groups," Communications in Algebra, vol. 34, no. 5, pp. 1691-1697, 2006.

[10] K. Wang and J. Guo, "Lattices generated by orbits of totally isotropic flats under finite affine-classical groups," Finite Fields and Their Applications, vol. 14, no. 3, pp. 571-578, 2008. 
[11] K. Wang and Z. Li, "Lattices associated with vector spaces over a finite field," Linear Algebra and Its Applications, vol. 429, no. 2-3, pp. 439-446, 2008.

[12] K. Wang and J. Guo, "Lattices generated by two orbits of subspaces under finite classical groups," Finite Fields and Their Applications, vol. 15, no. 2, pp. 236-245, 2009. 


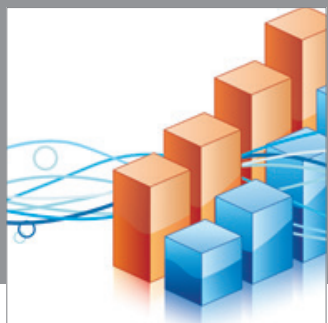

Advances in

Operations Research

mansans

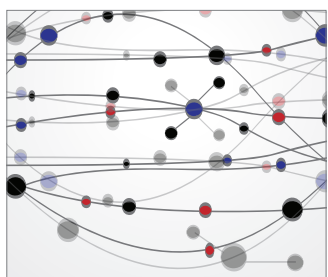

The Scientific World Journal
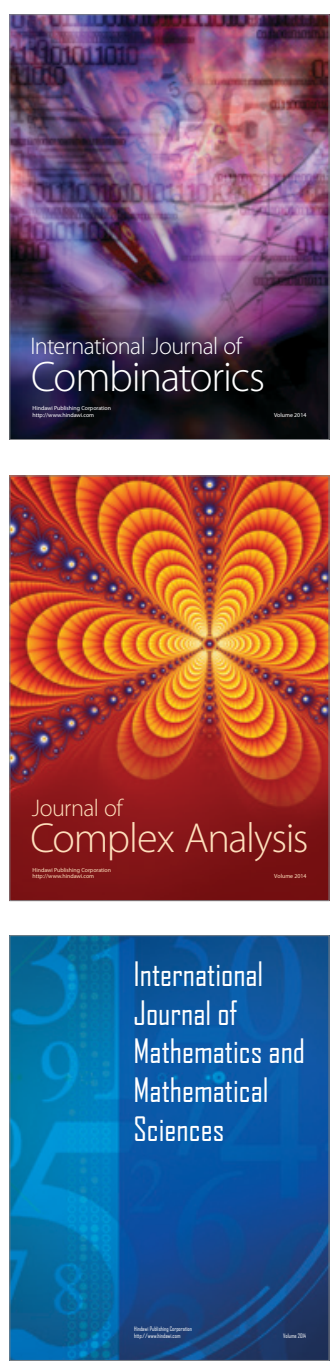
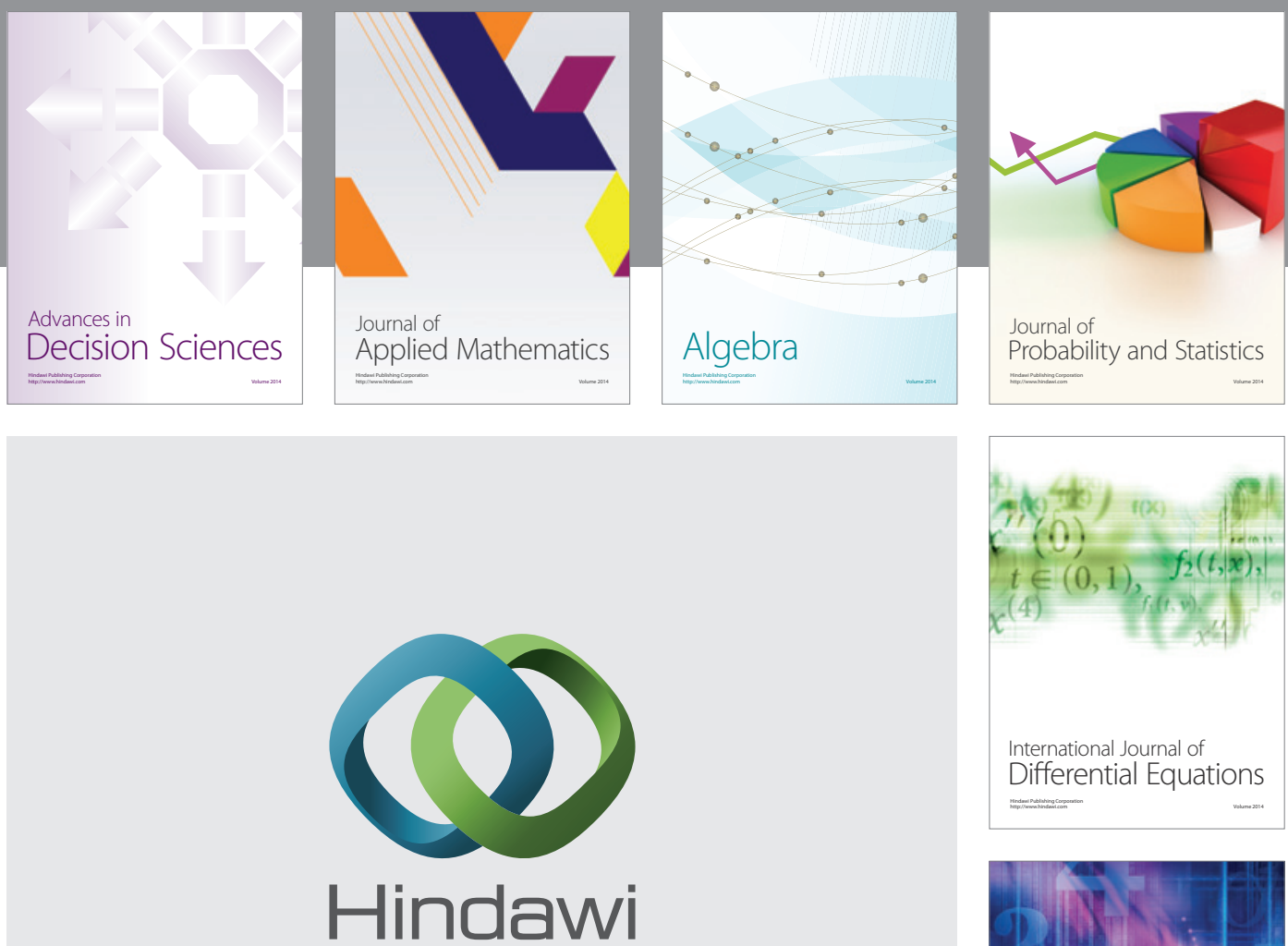

Submit your manuscripts at http://www.hindawi.com
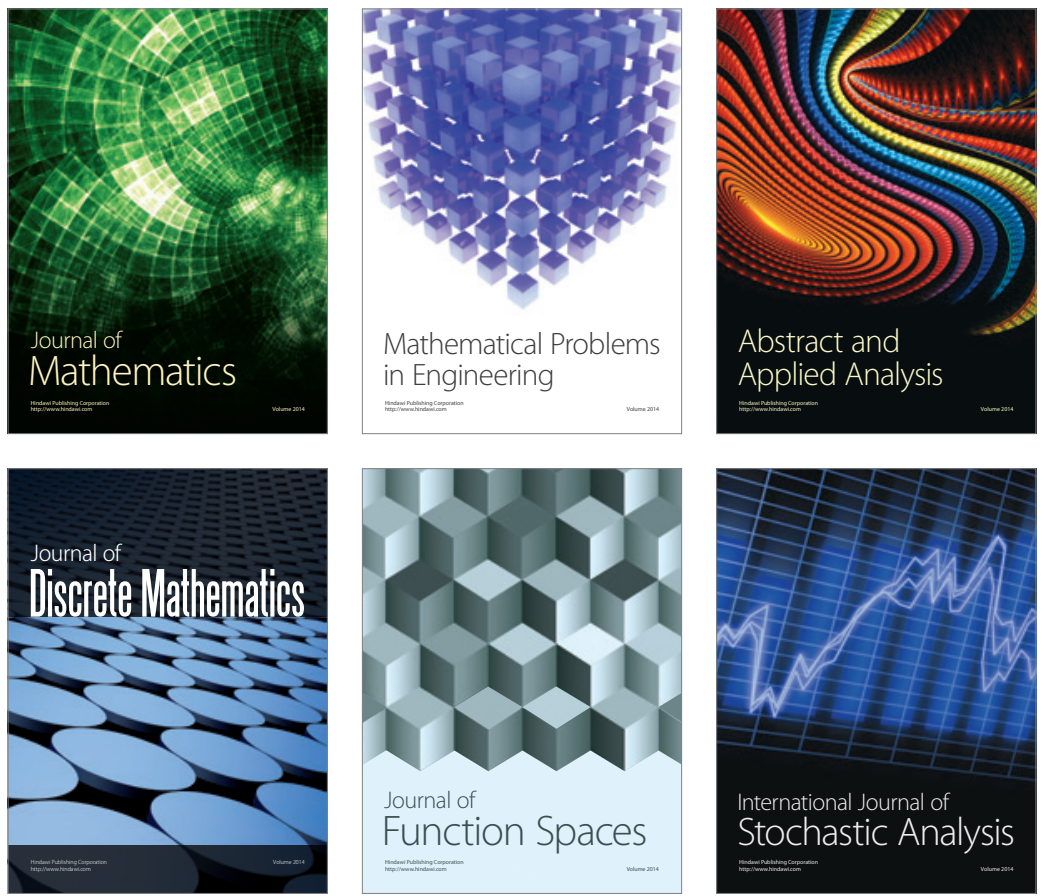

Journal of

Function Spaces

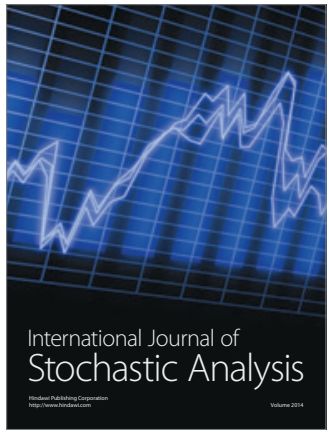

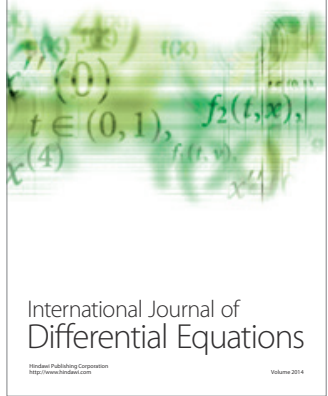
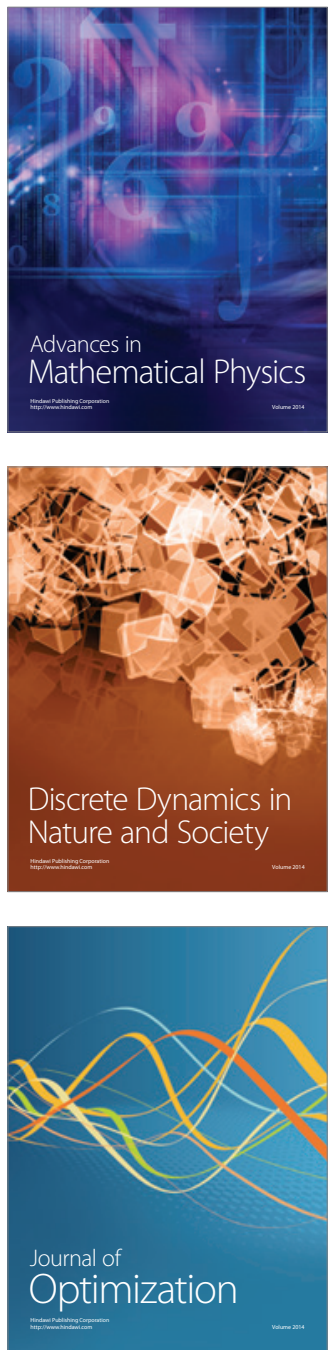